\title{
Development of Intelligent Traffic Control System based on Internet of Things and FPGA Technology in PROTEUS
}

\author{
Haoyuan $\mathrm{Ou}$ \\ Department of Information Engineer \\ Guangdong Polytechnic \\ Foshan, China \\ Jianming Zhang \\ Department of Information Engineer \\ Guangdong Polytechnic \\ Foshan, China
}

\author{
Yi Wang* \\ Department of Information Engineer \\ Guangdong Polytechnic \\ Foshan, China \\ wangyifsfz@163.com \\ * Corresponding Author
}

\begin{abstract}
In this paper, the research status of the intelligent traffic control system is analyzed, and the shortcomings of the current intelligent transportation system are pointed out. And the support technology of RFID technology, sensor network, communication and other supporting technology is proposed, which can construct the intelligent management platform of urban ground transportation. In this paper, the intelligent traffic control system is designed based on FPGA technology. Finally, the system is simulated by PROTEUS software. The simulation results show that the system works well.
\end{abstract}

Keywords- Intelligent Traffic Control System; FPGA; Internet of Things; PROTEUS; RFID

\section{INTRODUCTION}

With the development of economy, there are some problems which are difficult to solve in the transportation. The road congestion is becoming more serious, and the economic loss is more and more big. Now the traffic system has been unable to meet the needs of economic development [1]. Because of the improvement of living standards, people have put forward higher requirements on the safety and service level of transportation. In the traffic management of the introduction of SCM traffic light control instead of the traffic management personnel at the intersection service can help us to improve transportation safety, improve traffic management service quality. And to a certain extent, the economic losses caused by road congestion, but also reduce the labor intensity of the workers.

Traffic system is a complex system with random, fuzzy and uncertainty, and it is very difficult to establish a mathematical model, and sometimes it can not be described by the existing mathematical methods. At present most of the use of adaptive signal control, it needs the mathematical modeling, and do not consider the traffic delay, parking number, etc. So the classical control method is very difficult to get satisfactory results. The intelligent control is a control method without the establishment of a mathematical model, which can imitate the experience of the traffic police to control the traffic, to achieve a good control effect. There is no traffic congestion in the big cities all over the world. Traffic safety problem has become an important factor in the construction of harmonious society. Road traffic system is also facing a great challenge; the road traffic safety situation is extremely serious.

Relative to the previous loop coil and video as the main means of vehicle flow detection and so on the passive traffic control, the intelligent transportation of things, comprehensive coverage of the information collection, dynamic, intelligent control and other aspects. Through the integration of information and traffic information and feedback, the RFID, GIS, GPS and other technologies are integrated, and the only way to realize the information space is the vehicle.

FPGA/CPLD chip in the factory have done one hundred percent of the test, do not need to design personnel to bear the risk and cost of the chip, the design staff only in their own laboratory can be done through the relevant software and hardware environment to complete the chip design [2]. So, FPGA/CPLD's capital investment is less, reducing the potential cost.

The Internet of things is a network which is connected with human, human and material, by means of radio frequency identification and sensor. The main characteristics of the system can be used to analyze and process the information, and to improve the perception ability and realize the intelligent decision and control. At this stage, the application is mainly for the sensor network.

Intelligent transportation is a kind of real-time, accurate, and efficient and high technology integrated organization management and operation service system, which integrates the advanced information technology, communication technology, control technology and 
artificial intelligence technology and transportation organization to effectively integrate the organization management and service system. This paper proposes the use of the Internet of things technology and FPGA in the PROTEUS platform to develop intelligent traffic control system.

\section{INTERNET OF THINGS TECHNOLOGY APPLIED IN INTELLIGENT TRANSPORTATION}

China's vehicle quantity is increasing, traffic control is more and more, the use of computer instead of man to carry out efficient traffic management is the inevitable development trend, and let the computer control of traffic lights have a similar human perception intelligence, has a very strong practical significance, such as through the camera to make the traffic light control system to obtain visual perception function, can replace human eyes, make the system according to "see" traffic situation adaptive change control strategy, improve the traffic management automation level, make the traffic more efficient, more smoothly.

The development of urban road traffic automatic control system is based on the technology of urban traffic signal control and the development of the automobile industry. In its various stages of development, because of the various contradictions of the traffic, people always try to use the latest scientific and technological achievements of the various historical stages to the traffic automatic control, thus promoting the continuous development of automatic control technology.

Electronic bus stop board, the difference from the usual in the bus station to see the "iron" stop, is in the form of electronic or LCD screen display of bus information signs. Passengers in the station waiting for a bus, can see under a parked car and even the line all car driving through the electronic bus stop board, that is to say, it can tell you to wait for the bus will now travel to the station, from the station there are about how much time and distance, personnel on the car crowded degree, so that passengers in a timely manner to select the appropriate way to travel, evacuation traffic flow, improve the efficiency of road use. According to the actual situation, but also in the electronic bus stop board see brief news, weather forecast, traffic information, emergency announcement, video advertising instant messages, make people waiting for the bus is no longer a mood of anxiety.

Things technology and industry development will trigger a new round of information technology revolution and the industrial revolution, is the future of the field of information industry and industry upgrading of the commanding heights of the core driving force. China has started the research of the Internet of things in the world more than a decade ago, the technology and the standard and the international basic synchronization. Through a number of key technologies, the formation of a certain industrial scale and it is in the international standards for the development of a certain discourse. Intelligent transportation, intelligent security, intelligent logistics, public safety and other areas of demonstration in Beijing, Shanghai, Guangdong, Zhejiang and other provinces and cities have been initially launched. The development of the Internet of things will promote the process of intelligent.
Things are not just a concept, but a concept, has been in many industries to show the prototype of things.

ITS architecture has the following significance: ITS, it is more complex, involving a wide range, need to have a guiding framework to help us understand the structure of the system. ITS is a huge system, which includes a lot of subsystems, as is shown by equation (1). ITS architecture provides a unified interface standards for the various parts of ITS', so that all parts are easy to coordinate and integrate into a whole [3].

$$
E\left\{w(k) w^{T}(j)\right\}=Q(k) \delta_{k j}
$$

Through traffic information comprehensive real-time acquisition, the wireless transmission, data fusion, mathematical modeling, artificial intelligence and other technology, combined with GIS system for police, traffic jam warning, bus priority, public vehicles and special vehicles optimal path planning, dynamic induction, green wave control and emergency traffic control functions. Through the network flow analysis prediction and traffic status judgments, for network construction and traffic control strategy adjustment, transport planning to provide decision support and feedback.

Construction of advanced public is transportation system. Construction of special (bus, taxi, bus, ambulance, fire) vehicle monitoring and it is communication system. The monitoring center can receive and display the position, speed and direction of each vehicle in real time. The construction of public transportation planning and scheduling optimization platform; in a number of important bus lines set electronic bus stop board, to waiting passengers sign near the vehicle arrival information; to build the bus information query system, for the masses at home or in public places using individual or communal terminal bus route information obtained from the Internet [4]. Construction of advanced public transport system, and it is science and guidance of public travel and transfer, as is shown by equation (2).

$$
\gamma_{\mathbf{x}_{i} \mathbf{x}_{j}}=E\left\{\left[\mathbf{x}_{i}(t)\right]\left[\mathbf{x}_{j}(t+\tau)\right]^{T}\right\}
$$

The traffic network is the artery of the city, which symbolizes the industrial civilization level of a city. Transportation is related to people's interests in property, safety and time. With the excellent science of traffic control technology to the resources and logistics and people are very valuable, to ensure the smooth flow of traffic routes, in order to ensure smooth travel, logistics on time, and even the extension of life channel.

The construction of ITS system structure must first define the user of the system. As a branch of the information technology (IT) system, ITS can be defined as an IT system to define its users. The user of the information system is the influence of the system or the system of people and organizations can be from four aspects to identify the user of information technology system, namely: the need for IT, manufacturing IT, the use of IT and IT.

Now the bus stop mostly the function is single, available information is limited, for passengers, no 
intuitive vehicle arrival information as a reference to travel, for the management center, there is no visual real time vehicle distribution map to make the unified dispatch and management, in the era of high-speed information, to the public transportation intelligent showed urgent demand. In order to solve the above problems, the system to "for station passenger service" as the starting point and the ultimate goal in the electronic bus stop board display all the information provided for passengers, namely stop display module; at the same time, in order to effectively with the existing traffic information resources integration, in line users also provide consulting services; system will be a wealth management center of the electronic sign of the bus stop all management functions, convenient administrator of the system control and real-time scheduling [5].

$$
\bigcap_{j \in z} V_{j}^{2}=\{0\}, \overline{\cup_{j \in z}^{2}}=L^{2}\left(R^{2}\right),
$$

As an important part of the Internet of things industry, intelligent transportation has a high degree of market maturity, industry sensing technology mature, the government support strength, in the construction of digital city and smart city slogan, intelligent transportation systems in many cities have begun large-scale application, the market prospects, huge investment opportunities, will become the focus of the future years of things industry development. Things in the field of intelligent traffic have been a prototype, such as "car - Road" information system has been the focus of intelligent transportation development.

Traffic command center information platform plays a role in traffic information fusion, intelligent disposal, and information analysis and information distribution in the dynamic traffic information guidance system. The main functions of the information platform of the traffic command center: the collection and standardization of the floating vehicle traffic information collection system, the stationary traffic information collection system, the vehicle and the police officer positioning system.

Use of advanced traffic monitoring system to achieve vehicle parking fees, and it is improve highway capacity, maintain traffic safety. To establish an automatic detection and emergency rescue system for abnormal events, and to strengthen the automatic detection and management of the overload and speeding vehicles. Using information technology (database, geographic information system, and network) to improve the road construction, maintenance and infrastructure management, improve the management level, and reduce management and operating costs.

\section{DESIGN OF INTELLIGENT TRAFFIC LIGHT CONTROL SYSTEM BASED ON FPGA TECHNOLOGY}

In the FPGA, a large number of resources can be realized, which can accomplish the design of large scale combinational logic circuits, while a considerable number of storage circuits (flip flops) can also be completed in complex sequential logic circuits. By using various EDA tools, using the principle diagram or hardware description language, it is easy to realize the complex circuit in FPGA. Like a typical digital system frequency divider, digital clock, digital frequency meter, and so can be completed by FPGA.

Urban traffic control system is one of the components of transport system Intelligent, which is mainly used for the control and management of urban road traffic. Implementation of urban intersection traffic signal control is one of the basic signs of modern urban traffic management, is an important means to enhance the efficiency of traffic management. Signal controller is an important part of traffic signal control. All kinds of traffic control scheme, finally, the signal controller to achieve the intersection [6]. In order to ensure that the intersection of pedestrians and vehicles smoothly and smoothly through, often using electronic control of traffic signals to conduct command.

$$
\phi_{\mu}=\frac{\pi \mu}{8}
$$

The intelligent transportation system emphasizes on the system, information exchange and service. Its core technology is electronic technology, information technology, communication technology, traffic engineering and system engineering.

At present, there are many methods for designing the traffic lights, which are applied to the design of traffic signal controller, and the design of traffic light control system based on PLC is realized by CPLD. At present, the domestic traffic lights are generally located in the cross Lumen, prominently with red, green, yellow three colors of lights. Plus a countdown timer is to control the traffic. For the general situation of safe driving, vehicle diversion is still able to play a role, but according to the actual situation in the process, there are the following disadvantages: two lane of the vehicle in turn release time and fixed, at the crossroads, often a lane road, car more, release time should be longer; the other lane is a sub trunk, fewer vehicles, release time should be shorter. 2 did not consider the emergency vehicles through, the two lane should take measures, such as, fire trucks to perform emergency tasks through, the two lane should be stopped, so that emergency vehicles through.

8255 programmable parallel interface chip has three input and output ports, namely A port, B port and $\mathrm{C}$ port, corresponding to pin PA7 PB7, PA0 PB0 and PC7 $\mathrm{PC} 0$. There is also a control register inside the control port. Usually A port and it is B port as the input and output data port. C port as a control or status information port, it can be divided into 4 ports; each port contains a 4 bit latch. They are used in conjunction with port B / A, and can be used as a control signal output or as a state signal input.

FPGA is a high density of PLD, the integration of up to 35460 more than the door [7]. Figure 1 is a FPGA Xilinx, which consists of three programming elements and a static memory for storing programming data. The three programmable elements are IOB (Input / Block Output programmable input / output unit), CLB (Logic Block Configurable programmable logic unit) and IR (Resource Interconnect resource). Their working state is all set by the data in the programming memory. CLB provides the logic functions that a user needs. Since these three parts are programmable, so the function of the chip can be changed 
by changing the connection between the CLB, but also by changing the logic functions of the CLB.

$$
\begin{aligned}
& x_{k}=f\left(x_{k-1}, u_{k-1}, w_{k-1}\right) \\
& z_{k}=h\left(x_{k}, v_{k}\right)
\end{aligned}
$$

In addition to the system according to the vehicle flow automatically control adjustment, can also be manually set by the keyboard, increase the artificial controllability, to avoid automatic failure and accident, and then the emergency state, can be set up all the lights for a red light. Keyboard is the most commonly used in the single chip microcomputer system interface, the general situation of two kinds of independent and determinant. The former software writing is simple, but the number of keys in a lot of special waste I / 0 port resources, the number of keys is generally less than the number of systems. The latter applies to the number of keys, but in the microcontroller I / 0 port resources are relatively small and need more keys; this method can not meet the design requirements. The system requirements of the key control is not much, and I / 0 is enough, can be directly used in independent.

AT89C51 is a 4K FPEROM and Flash Programmable Erasable Read Only Memory, which uses a static CMOS process to produce 8 bit microprocessors, the highest operating frequency 24MHZ. AT89C5 configuration and pinot as shown in Figure 1.

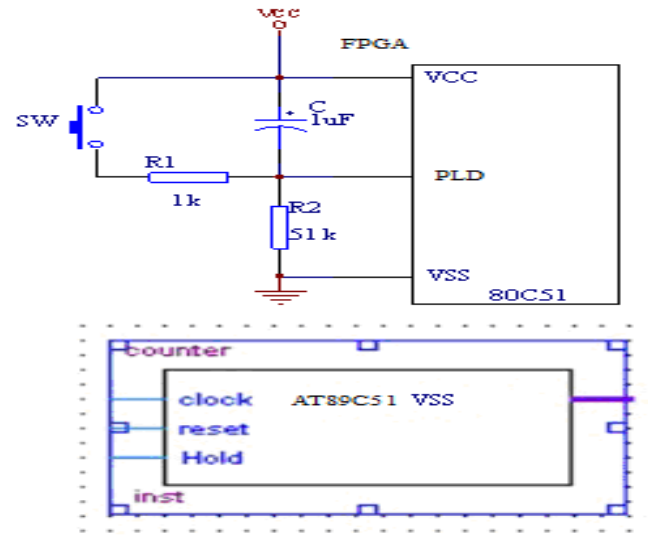

Figure 1. FPGA Technology and AT89C5 Configuration and Pinout.

RST: reset input. When the oscillator reset device, to maintain the high level of the RST pin two machine cycle. P0 port: $\mathrm{P} 0$ port is an 8 bit open circuit two-way I/O port, each foot can absorb 8TTL gate current. When the P1 pin is first written 1 , it is defined as the high impedance input. P0 can be used for external program data memory, which can be defined as the data / address of the eighth [8]. In flash programming, $\mathrm{P} 0$ port as the input port of the original code, when flash check, P0 and outputs the code bytes. At this time $\mathrm{P} 0$ external must be pulled high.

Design input consists of three types of hardware description language HDL, state diagram and schematic diagram. HDL design method is a good form of the modern design of large-scale digital integrated circuits, in addition to the IEEE standard VHDL and HDL Verilog two forms, there are FPGA manufacturers to launch a special language, such as Quartus AHDL. HDL language description in the state machine, control logic, bus function, the circuit can be described by specific Compiler (such as Synopsys II FPGA or Express FPGA) to achieve the specific hardware module, and the principle diagram of the top design, data path logic, manual optimization circuit and other aspects of the Quartus Altera software environment, you can use Editor Momory to edit the internal memory [9]. The common way is to HDL language, the principle diagram is supplemented, carries on the mixed design to display the two respective characteristic. In general, the FPGA vendor software with third party software is equipped with interface; you can import the third party design document processing.

ALE/PROG: when accessing external memory, the address latch allows the output level to be used to latch the address of the byte. During the FLASH programming, this pin is used for the input of the programming pulse. At ordinary times, the ALE is the same as the constant frequency of the output pulse signal, the frequency of the oscillator frequency of $1 / 6$. So it can be used against external output pulse or for timing purposes. However, it should be noted that when the external data memory is used, a ALE pulse is skipped. If you want to prohibit the ALE output can be in SFR8EH address for 0 .

Vehicle security configuration information equipment, rear end collision warning and collision warning and vehicle driving state records and satellite positioning, electronic map navigation system, passenger counting device, electronic fare collection equipment. Using related technologies such as RFID and weight sensor through the vehicle recognition, vehicle recognition, license plate recognition, dynamic said again to vehicle safety management.

\section{DEVELOPMENT OF INTELLIGENT TRAFFIC CONTROL SYSTEM BASED ON INTERNET OF THINGS AND FPGA TECHNOLOGY IN PROTEUS}

The application of the Internet of things is not only reflected in the identification, but also to play the advantages of its network. Based on the concept of RFID and other label technology is also beginning to be common, Volkswagen and other car manufacturers. "Car networking" concept is more able to reflect the application of RFID and other things technology. "Car networking" means the electronic tags loaded on the vehicle through the RFID and other identification technology, the information network platform for all vehicles on the property information and static, dynamic information extraction and effective use, and according to different functional requirements of all vehicles running state to effectively monitor and provide integrated services. The state is promoting the development of automotive digital standard source technology. Automotive digital standard source technology is based on the RFID development of information resources related to the application of information technology, it will promote the development of the car networking and RFID industry process.

The basic factors that affect the road are: people, cars, roads, environment, and these four basic elements. Intelligent transportation system should be divided into the following main functions: first, the information detection and sensing system. The real-time traffic flow of road vehicles is detected by means of magnetic, RFID, GPS and other sensors. The real-time speed of vehicles is monitored 
by means of radar and other sensors [10]. By using the RFID technology and sensor technology to obtain the information of the state information of the object, the physical world and the virtual world are established. Sensor information acquisition information through the Internet, $3 \mathrm{G}$ or other ways is to send data to the data processing center, the composition of large-scale networks, as is shown by equation (6).

$$
S_{\mathbf{x}_{i} \mathbf{x}_{j}}=P_{x_{i} x_{j}}+i Q_{x_{i} x_{j}}=S_{x_{i}} \times S_{x_{j}}^{*}
$$

Through the analysis, we can know that the design of the crossroads traffic lights control circuit to be able to make the north and south, East and West four lights (red, yellow, green, left), the four lights can be sequentially lit. And requires a green light to turn red light or turn left turn lights before you turn yellow lights for 5 seconds, turn left before the lights turn red lights turn yellow light 5 seconds, red light can be directly to the green or turn left lights. Four kinds of lights are also required to be able to light the time to show up in the form of a countdown. The rational design of system functions with VHDL language may to red, yellow, and Green left turn lights conversion an accurate time interval and the switching sequence.

PROTEUS embedded system simulation software in the design has been noted and the integration of various compiler procedures, such as it can Keil, Wave6000, and so on. Due to the use of Wave6000, and it is with a strong software simulation and hardware simulation. The combination of Proteus and Wave6000 to debug the hardware is more convenient, here is the use of Proteus+Wave6000 simulation method, the specific steps are as follows: first run VSM ISIS PROTEUS, select Source Define Code Generation Tool menu item, will appear as Figure 2 defines the code generation tool dialog box.

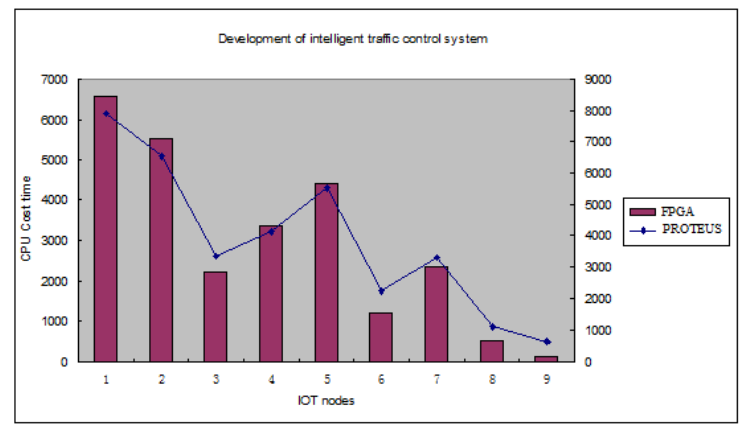

Figure 2. Comparison of Intelligent Traffic Control System Based on Internet of Things and FPGA Technology in PROTEUS.

After the completion of the circuit diagram, and then add the AT89C51 application. Will the mouse cursor over the AT89C51, click the right mouse button to in the selected state, left click on the device, in the program file bar add compiled hex format program files AA.hex (can accept three kinds of file format), to the AT89C51 input crystal oscillator frequency, where the default $12 \mathrm{Mhz}$. Click OK button to complete the program to add work, the following can be system simulation is carried out. Click the button below the main interface to start the system simulation. VSM PROTEUS is a kind of interactive simulation, in the simulation of the control buttons, switches and other operations, the system's response to the input will be reflected in the real as shown in figure 2.

Through networking RFID, sensor networks, shift communication support technology, urban construction ground traffic intelligent management platform, including center city traffic, real-time monitoring and dynamic induction system, fixed-point measurement system of the motor vehicle, Chuang ban vehicle intelligent snapping system and traffic signal lamp intelligent control system and other subsystems. At the same time, it can also construct the intelligent guidance and management system of the parking lot.

\section{SUMMARY}

This paper presents the development of intelligent traffic control system based on Internet of things and PROTEUS technology in the FPGA platform. The system takes SCM as control core; the system is connected to the minimum system, which is composed of the traffic detection module, the detection module, and the key module. Intelligent traffic signal control system uses three layers of distributed structure, signal machine through the RS232/RJ45 and the central link, using RJ45 network port form. The system structure is divided into three layers: signal control center, communication section and intersection part.

\section{REFERENCES}

[1] Ming-Huo Chen, Chao-Lung Ting, Ray-I Chang, "Safe Driving Assistance by Lane-Change Detecting and Tracking for Intelligent Transportation System", IJIPM, Vol. 4, No. 7, pp. 31 38, 2013

[2] Thirumalai T., Kashwan K. R., "Split Ring Patch Antenna for FPGA Configurable RFID Applications", JNIT, Vol. 6, No. 3, pp. $47 \sim 58,2015$.

[3] Ke Zheng, KeCheng Liu, "The research of intelligent transportation system based on formal concept ontology models", JCIT, Vol. 7, No. 10, pp. $158 \sim 165,2012$.

[4] Zhang Qigui, Zhao Lijuan, "Implementation of Bayer Image Interpolation based on FPGA", JNIT, Vol. 4, No. 1, pp. $19 \sim 24$, 2013.

[5] XianFeng Yang, Zhen Liu, "The Application of Association Rules Mining in Building Intelligent Transportation Systems", JCIT, Vol. 7, No. 20, pp. $575 \sim 582,2012$.

[6] Md. Motaharul Islam, Pham Phuoc Hung, Al - Amin Hossain, Mohammad Aazam, , "A Framework of Smart Internet of Things based Cloud Computing", RNIS, Volume 14, pp. $646 \sim 651,2013$.

[7] Hao Wang, "Wireless Sensor Networks for an Extended City Intelligent Transportation System", IJACT, Vol. 3, No. 5, pp. 300 307, 2011.

[8] Xiaoxia Niu, Yanxia Wu, Bowei Zhang, Guochang Gu, Guoyin Zhang, "Improved FPGA-Based Area Estimation Method for Hardware/Software Partitioning", JCIT, Vol. 8, No. 4, pp. 636 643, 2013.

[9] Krishan Sabaragamu Koralalage and Noriaki Yoshiura, "OTag: Architecture to Represent Real World Objects in RF Tags to Improve Future Intelligent Transportation Systems", JCIT, Vol. 4, No. 2, pp. $30 \sim 48,2009$

[10] Hua-Mei Xin, Kun Yang, "A Routing Protocol for Internet of Things with High-speed Mobile Nodes", IJACT, Vol. 5, No. 7, pp. $197 \sim 205,2013$ 